\title{
High-bootstrap, noninductively sustained electron internal transport barriers in the Tokamak à Configuration Variable ${ }^{a)}$
}

\author{
S. Coda, ${ }^{\text {b) }}$ T. P. Goodman, M. A. Henderson, O. Sauter, R. Behn, A. Bottino, \\ Y. Camenen, E. Fable, An. Martynov, P. Nikkola, A. Scarabosio, G. Zhuang, \\ C. Zucca, and the TCV team ${ }^{\text {c) }}$ \\ Ecole Polytechnique Fédérale de Lausanne (EPFL), Centre de Recherches en Physique des Plasmas, \\ Association Euratom-Confédération Suisse, EPFL SB CRPP, Station 13, CH-1015 Lausanne, Switzerland
}

(Received 22 November 2004; accepted 1 March 2005; published online 5 May 2005)

\begin{abstract}
Important ingredients of the advanced-tokamak route to fusion have been explored in depth in the Tokamak à Configuration Variable [F. Hofmann, J. B. Lister, M. Anton et al., Plasma Phys. Controlled Fusion 36, B277 (1994)] over the past two years. Using a uniquely powerful and flexible electron-cyclotron resonance heating (ECRH) system as the primary actuator, fully noninductive, steady-state electron internal transport barrier discharges have been generated with an electron-energy confinement time up to five times longer than in $L$ mode, poloidal $\beta$ up to 2.4 , and bootstrap fraction up to $75 \%$. Interpretative transport modeling confirms that the safety factor profile is nonmonotonic in these discharges. The formation of the barrier is a discrete event resulting in rapid and localized confinement improvement consistent with the time and location of magnetic-shear reversal. In steady state, however, the confinement quality appears to depend on the current gradient in a broader negative-shear region enclosed by the barrier, improving with increasing shear: in particular, the width and depth of the barrier can be controlled and finely tuned, along a magnetohydrodynamic-stable path, by manipulating the current profile with ECRH (six independently steerable $0.45 \mathrm{MW}$ launchers). The crucial role of the current profile has been clearly demonstrated by applying small Ohmic current perturbations which dramatically alter the properties of the barrier, enhancing or reducing the confinement with negative and positive current, respectively, with negligible Ohmic heating. These results are in agreement with theoretical estimates: first-principle-based numerical simulations of microinstability dynamics and turbulence-driven transport predict a substantial suppression of turbulence and anomalous energy diffusivity near the location of the minimum in the safety factor. (c) 2005 American Institute of Physics. [DOI: 10.1063/1.1896953]
\end{abstract}

\section{INTRODUCTION}

The ideal path towards a commercially viable nuclearfusion reactor involves the simultaneous optimization of several physical properties of the reacting fuel. Within the real constraints of scientific experimentation, different avenues are being explored in parallel by the fusion community, placing special emphasis on one or the other of those properties. Foremost among these properties is the confinement of the plasma, i.e., its ability to retain the physical quantities that are provided externally to maximize its reactivity, such as energy and particle content. The improvement of energy confinement in magnetic-confinement devices, in particular, requires a reduction of cross-field transport towards the ultimate limit determined by Coulomb collisions. In the past two decades, considerable progress has been made along this avenue, mostly through the establishment of plasma discharge conditions leading to the appearance of localized regions of highly reduced energy diffusivity, effectively acting as transport barriers. This can occur either at the edge of the plasma

\footnotetext{
a) Paper RI1 6, Bull.Am.Phys.Soc. 49, 326 (2004).

${ }^{b)}$ Invited speaker. Electronic mail: stefano.coda@epfl.ch

${ }^{c)}$ See the Appendix.
}

$\left(\mathrm{H}_{\text {mode }}{ }^{1}\right)$ or in its core [internal transport barriers or ITBs (Ref. 2)], and double barriers have also been observed in several cases. In the highest performance instances of such scenarios in tokamaks, the energy confinement of the ion component has approached the collisional Coulomb level, ${ }^{3}$ known in this context as neoclassical confinement.

An additional desirable property of a fusion power plant would be its ability to run continuously, rather than in a pulsed fashion, as is the case with all existing tokamak experiments. This would require the abandonment of the transformer principle for generating the necessary toroidal electric current, which instead would be supplied by noninductive means, such as externally launched electromagnetic waves ${ }^{4}$ or the internal neoclassical bootstrap current driven by the plasma pressure gradient.

Key ingredients in the generation of transport barriers are the details of the current-density and flow-velocity profiles, which have a complex relationship with a variety of externally controlled quantities such as the plasma density and shape, the toroidal magnetic field, and the net input heating power. The pursuit of conditions leading to enhanced performance compatible with steady-state operation, particularly, with a large bootstrap current fraction, has become 
known as the advanced tokamak program. ${ }^{5}$ Within this set of activities, interest has shifted more recently to the confinement properties of the electron fluid. ${ }^{2,6,7}$ This has direct relevance to a future D-T reactor, in which energy from energetic $\alpha$ particles will be preferentially transferred to electrons and the electron temperature $T_{e}$ is expected to exceed the ion temperature $T_{i}{ }^{8}$ In addition, anomalous electron transport (i.e., the ratio of the experimental to the neoclassical diffusivity) is typically approximately one order of magnitude higher than anomalous ion transport, ${ }^{8}$ and the need to find avenues for improvement is correspondingly greater. Considerable technological developments in high-frequency, highpower, long-pulse gyrotrons, ${ }^{9}$ which can heat electrons efficiently by electron cyclotron resonance heating (ECRH) and thus effectively simulate $\alpha$-particle heating, have opened the way to a rich field of study. Electron ITBs (eITBs) have been generated in several devices ${ }^{2,6,7,10-12}$ and a substantial body of work now exists, both theoretical and experimental, aiming to elucidate the physics of these phenomena and to shed light on both their similarities to and differences from ion ITBs.

Work on the Tokamak à Configuration Variable (TCV) (Ref. 13) in the past few years has contributed to research along the advanced tokamak path from a unique perspective. ${ }^{11,14,15}$ TCV plasmas are heated with secondharmonic ECRH at extremely high power densities (fluxsurface averages in excess of $30 \mathrm{MW} / \mathrm{m}^{3}$ ) and at low plasma density $\left(~ \leqslant 3 \times 10^{19} \mathrm{~m}^{-3}\right.$, dictated by ECRH accessibility). An important consequence of the low operational density is poor coupling between electrons and ions, which causes the electron temperature to be much larger than the ion temperature. The confinement properties of the electrons can thus be studied virtually in isolation, under extreme heating conditions, and in the absence of externally driven plasma flows. The ECRH delivery system provides unique flexibility: the second-harmonic system at $82.7 \mathrm{GHz}$ consists of six 0.45 MW beams delivered by six independent launchers, which can be steered in real time to provide either pure heating or cocurrent and countercurrent drive (ECCD) simultaneously at different locations within the plasma. ${ }^{16}$ An equally flexible control system, based on 16 independently powered shaping coils, permits the control of extremely varied plasma shapes and positions within the vacuum chamber (elongation 0.9 $\leqslant \kappa \leqslant 2.8$, triangularity $-0.6 \leqslant \delta \leqslant+0.9$ ). The main plasma parameters are as follows: major radius $R=0.88 \mathrm{~cm}$, minor radius $a=0.25 \mathrm{~cm}$, plasma current $I_{p} \leqslant 1 \mathrm{MA}$, toroidal magnetic field $B_{\phi} \leqslant 1.54 \mathrm{~T}$, total ECRH power (second and third harmonic, 82.7 and $118 \mathrm{GHz}$, respectively) $P_{\mathrm{EC}}=4.5 \mathrm{MW}$.

The versatility of ECRH for driving current, tailoring the current profile, and heating electrons in a localized fashion makes it an ideal tool for studies of enhanced-performance scenarios in steady-state conditions. Indeed, fully noninductive operation with ECCD and bootstrap current for times well in excess of the resistive current redistribution time $\left(\tau_{\mathrm{CRT}}\right)$ is routine on TCV. ${ }^{11,17,18}$ Improved core electron confinement was also obtained early on with ECRH and ECCD. ${ }^{11,14}$ As illustrated in Fig. 1, the two scenarios have progressively been merged with the generation of fully noninductively sustained eITBs with poloidal $\beta$ up to 2.4 , boot-

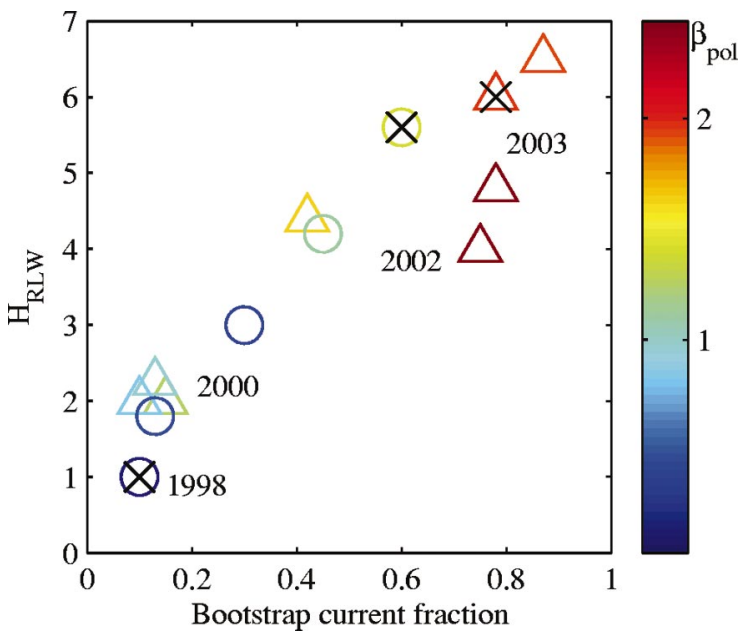

FIG. 1. (Color). Electron energy confinement enhancement factor over the Rebut-Lallia-Watkins scaling (TCV $L$-mode scaling) vs bootstrap current fraction for representative TCV discharges. The color coding refers to $\beta_{\mathrm{pol}}$. Triangles are for fully noninductive discharges and circles for discharges with an inductive component. The crosses indicate shots in which the highperformance phase lasted less than $2 \times \tau_{\mathrm{CRT}}$.

strap fraction exceeding $80 \%$, and energy confinement enhancement up to a factor of 6 over the TCV $L$-mode scaling. The latter two parameters have reached $75 \%$ and 5 , respectively, for durations exceeding $3 \times \tau_{\mathrm{CRT}} \cdot{ }^{15,19}$ (In this paper, the confinement enhancement will be characterized by the parameter $H_{\mathrm{RLW}}$, expressing the ratio of the electron energy confinement time $\tau_{e E}$ to that predicted by the Rebut-LalliaWatkins scaling. ${ }^{20}$ The latter is an excellent predictor of TCV $L$-mode confinement, which owing to the poor confinement properties of electrons is typically a factor of 2-3 lower than the ITER-L-98 scaling. ${ }^{8}$ )

The operational recipe for generating noninductive eITBs is as follows. ${ }^{15,19}$ The discharge is initiated as a canonical Ohmic discharge. After the beginning of the current flat top, co-ECCD is applied off-axis, between $\rho=0.2$ and 0.5 ( $\rho$ being a normalized radial coordinate proportional to the square root of the enclosed plasma volume). The externally applied electric field is then switched off by holding the current in the transformer primary constant. Stiff feedback control is used on the applied coil voltage in order to keep the coil current close to its preprogramed value, while control of the plasma current is relinquished. The formation of an eITB, signaled by the appearance of a localized steep pressure gradient, is observed after a time of the order of $\tau_{\mathrm{CRT}}$. Once the current profile evolution is complete, applying power in the low-diffusivity core region raises the global confinement rapidly, over a confinement time scale. A definition has been proposed to quantify the barrier strength, ${ }^{21}$ which is identified with the parameter $\rho_{\max }^{*}$, i.e., the maximum of $\rho_{T}^{*}$ $=\rho_{s} / L_{\mathrm{Te}}$, where $\rho_{s}$ is the ion sound gyroradius and $L_{\mathrm{Te}}$ is the electron temperature scale length, and all quantities are measured on the outer midplane. An example of the steady-state electron-pressure, power-deposition, and $\rho_{T}^{*}$ profiles is shown in Fig. 2, and the corresponding time evolution of the relevant quantities is depicted in Fig. 3. Numerous variations on this basic scenario or perturbations to it are of course pos- 


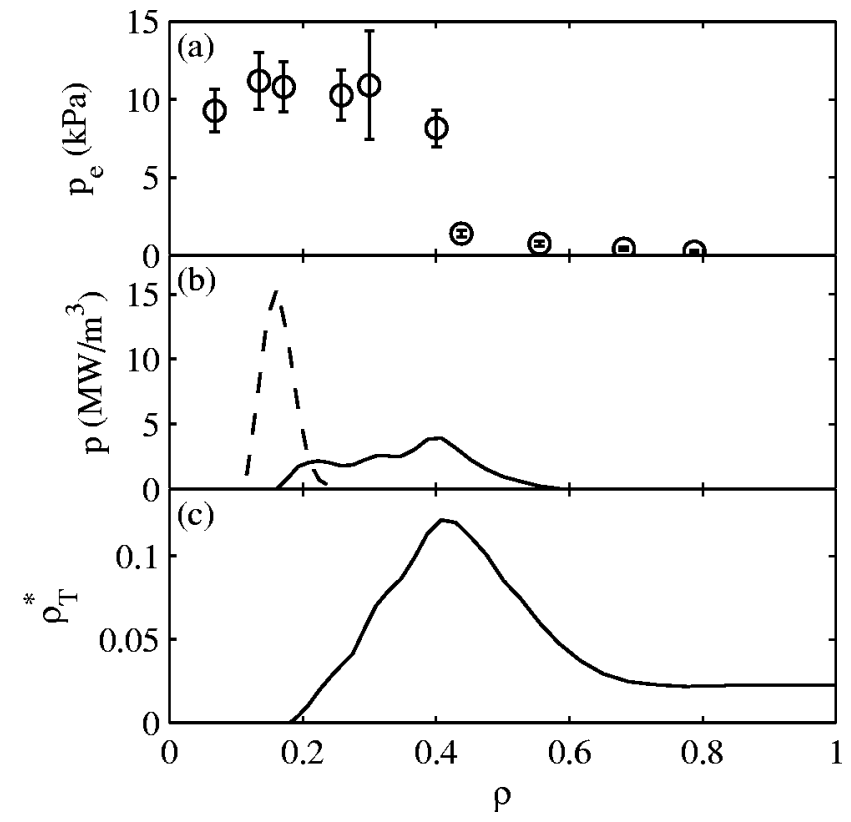

FIG. 2. (a) Electron-pressure profile from Thomson scattering; (b) ECRH power-deposition profile calculated by ray tracing (solid curve, off-axis coECCD sources; dashed, core counter-ECCD); (c) $\rho_{T}^{*}$ profile, for shot 25645 at $1.5 \mathrm{~s}$.

sible, and some of the more relevant ones for current research will be discussed in the remainder of this paper. Although TCV lacks a current-density $(j)$ profile measurement at present, a combination of indirect experimental measurements and numerical modeling ${ }^{19,22}$ suggests that the off-axis co-ECCD and the bootstrap current localized in the highpressure-gradient region combine to produce a hollow current profile and a nonmonotonic safety-factor $(q)$ profile. The bootstrap current is favored by the formation of the barrier, and a positive feedback loop is initiated that keeps the bootstrap current density well aligned with the $q$ profile required for the barrier, into the steady-state phase..$^{19,21}$

The lack of externally imparted angular momentum makes the generation of large-scale sheared flows unlikely, and suggests that $\mathbf{E} \times \mathbf{B}$ shear stabilization of turbulence does not play a role in the physics of these barriers. This remains to be confirmed as the radial electric field is not yet known in these discharges. Only the toroidal rotation has been measured thus far and has indeed been found to be negligible. ${ }^{23}$

The aim of this paper is to describe the current status of steady-state high-performance scenarios in TCV and of our theoretical understanding of the underlying physical mechanisms at play. Section II is concerned with the formation of the barrier. Evidence is presented to correlate the barrier formation, a rapid event, with the time and location of the appearance of a radial location of zero magnetic shear $s$ $=(\rho / q) d q / d \rho$. Once the barrier is formed and the current diffusion is complete, steady-state conditions are achieved, provided magnetohydrodynamic (MHD) stability is preserved. Steering a MHD-free course has proven operationally feasible by controlling both the $q$ and pressure profiles through the location, ECCD content, and power ratios of the off-axis and on-axis beams. The phenomenology of the eITB

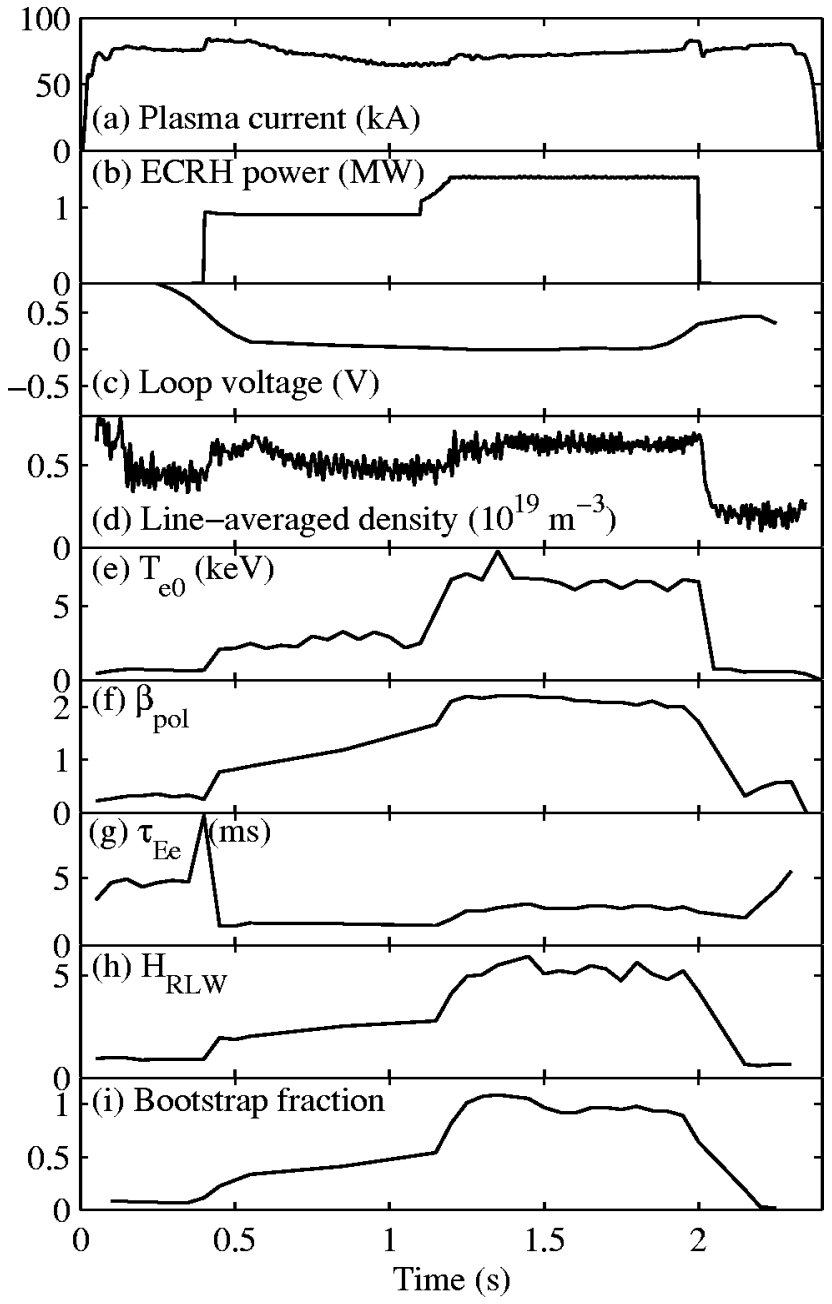

FIG. 3. TCV discharge 25645: (a) plasma current; (b) ECRH power; (c) loop voltage; (d) line-averaged density; (e) central electron temperature; (f) poloidal $\beta$; $(\mathrm{g})$ electron-energy confinement time; (h) electron-energy confinement enhancement over the Rebut-Lallia-Watkins scaling; (i) bootstrap current fraction.

in steady state is discussed in Sec. III. The barrier location remains constant over a time up to an order of magnitude longer than $\tau_{\mathrm{CRT}}$ and two orders of magnitude longer than $\tau_{e E}$.

The application of a small Ohmic current perturbation, accompanied by negligible energy transfer, to an otherwise fully noninductive eITB discharge has yielded a clear, unambiguous proof of the key influence of the core current profile on the properties of the barrier: a small amount of cocurrent substantially degrades the barrier while a small amount of countercurrent enhances it, both effects increasing with current. ${ }^{24,25}$ This demonstration will be the subject of Sec. IV. Section V presents an initial theoretical corroboration of the role of the current profile in the eITB physics; transport modeling is used to study the current profile evolution, and gyrokinetic and gyro-Landau-fluid simulations are performed to investigate the microinstability growth rates. Finally, Sec. VI concludes the paper with final remarks. 


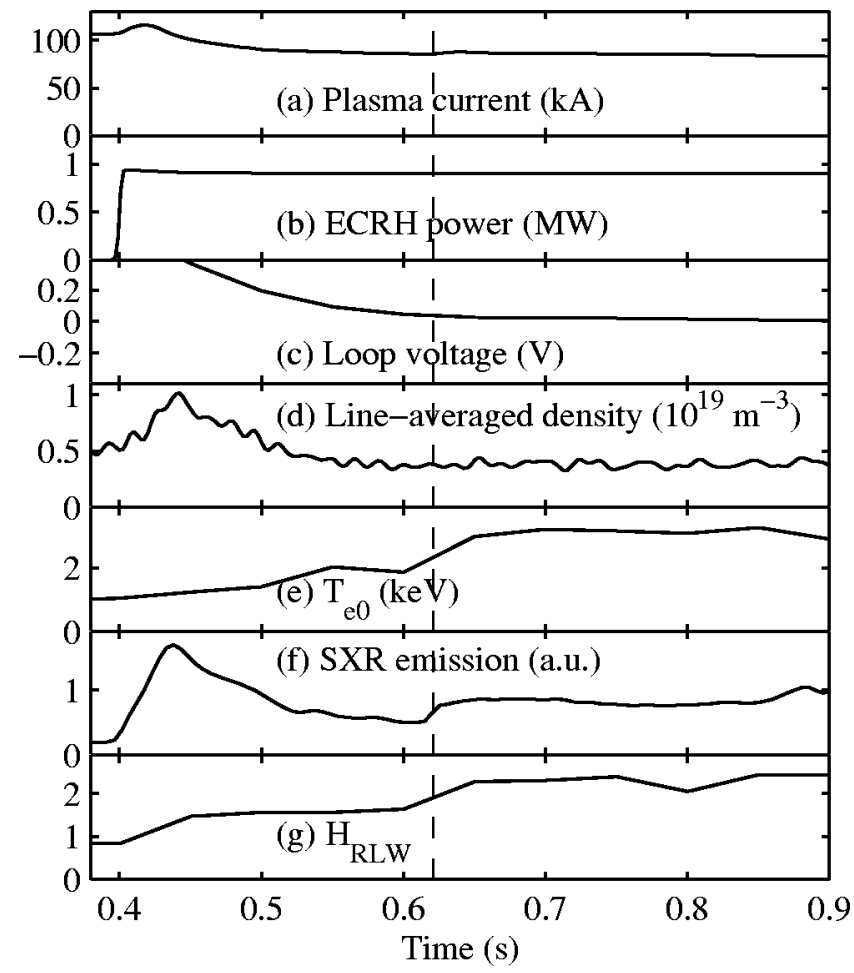

FIG. 4. TCV discharge 21655: (a) plasma current; (b) ECRH power; (c) loop voltage; (d) line-averaged density; (e) central electron temperature; (f) soft-x-ray emission from a central chord; $(\mathrm{g})$ electron-energy confinement enhancement over the Rebut-Lallia-Watkins scaling. The vertical dashed line indicates the time of the eITB formation.

\section{FORMATION OF THE EITB}

The standard operational scenario for generating a noninductive eITB involves a transition from an Ohmic, centrally peaked current-density profile to a hollow profile driven by off-axis current sources. ${ }^{15}$ The initial Ohmic current is designed to be close to the value that the noninductive sources are able to drive. In typical scenarios with two 0.45 MW beams aimed at $\rho \simeq 0.3$, we set $I_{p} \simeq 80-100 \mathrm{kA}$. A good match minimizes the evolution in the plasma configuration caused by the total current growth or decay, which occurs over a global $L / R$ time scale, which is equivalent to the time for current diffusion through the entire plasma column. The redistribution of the current profile from peaked to hollow involves a diffusion over only $\approx 1 / 3$ of the plasma radius, and the scaling of the diffusion time with the square of the distance results in a redistribution time $\tau_{\mathrm{CRT}}$ one order of magnitude smaller than the $L / R$ time. Experimentally $\tau_{\mathrm{CRT}}$ is estimated to be $\sim 0.2 \mathrm{~s}$, as will be seen later.

During the Ohmic phase the current penetration is rather rapid $(<0.2 \mathrm{~s})$, owing to the low temperature (typically $0.6-$ $0.8 \mathrm{keV}$ at this current), and is complete by the time coECCD is switched on. The temporal evolution of the plasma current, surface loop voltage, line-integrated electron density, central temperature, soft-x-ray emission from a centrally viewing chord $\left(I_{\mathrm{SX} 0}\right)$, and confinement enhancement factor after the co-ECCD switch-on is shown in Fig. 4, along with the ECRH power. In the time range considered in this figure, no central heating is applied and the current profile is still evolving. A clear upward step is seen in $T_{e 0}, I_{\mathrm{SX} 0}$, and $H_{\mathrm{RLW}}$

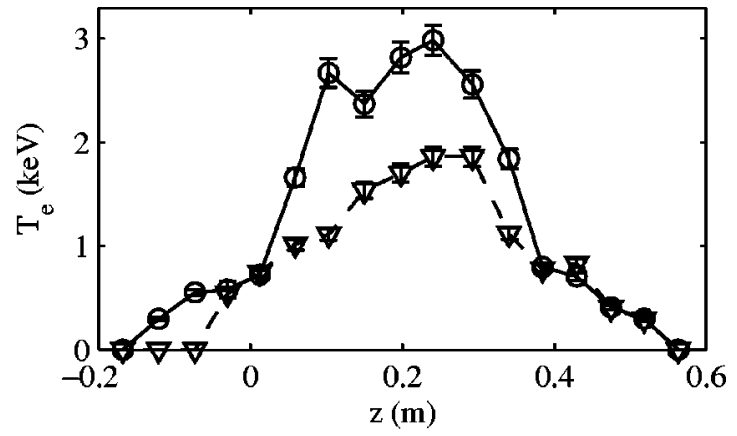

FIG. 5. Electron-temperature profiles from Thomson scattering along a central vertical chord for discharge 21655 at $0.6 \mathrm{~s}$ (triangles and dashed curve) and $0.65 \mathrm{~s}$ (circles and solid curve).

at $\sim 0.62 \mathrm{~s}, \quad$ signaling a sudden improvement in confinement. $^{26}$ This is borne out by the $T_{e}$ profiles measured by Thomson scattering before and after the transition (Fig. 5), which reveal the appearance of a barrier.

To analyze the details of the transition and determine its characteristic time, the temporal resolution of Thomson scattering $(50 \mathrm{~ms})$ is insufficient. We turn therefore to a measurement of vertically line-integrated soft-X-ray emission, performed with a multiwire chamber proportional detector with up to $5 \mu$ s time resolution and $\sim 1.2 \mathrm{~cm}$ radial resolution. This measurement lends itself optimally to an integral inversion in order to reconstruct the local emissivity. This procedure is performed under the assumption of poloidal homogeneity by employing the Fisher regularization algorithm. The result for one discharge is shown in Fig. 6. On average the inverted signal is slightly hollow, the result of a nearly flat central pressure profile and of a hollow effective charge $Z_{\text {eff }}$. The transition (at time $0.545 \mathrm{~s}$ in this discharge) is clearly seen to occur in less than $3 \mathrm{~ms}$, a time of the order of $\tau_{e E}$. The measured emissivity, taking into account the instrumental responsivity, varies roughly like $T_{e}^{1.4}$ in this temperature range, under the assumption that the density $n_{e}$ does not vary,

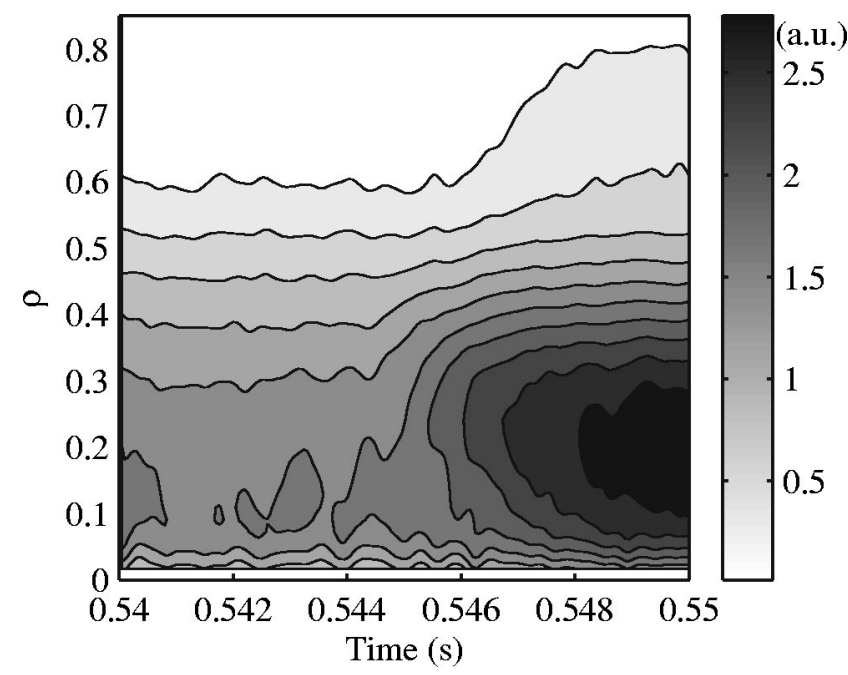

FIG. 6. Contours of local soft-x-ray emissivity derived by Fisher inversion from line-integrated measurements performed by a vertically viewing multiwire chamber proportional detector (TCV discharge 24914). The minimum $\rho$ resolved by the procedure is $\rho=0.017$. 
as suggested by Fig. 4 and as corroborated by local Thomson-scattering measurements, and that $Z_{\text {eff }}$ does not vary appreciably over the rapid time scale under study. Figure 6 shows that the increase in temperature, hence in confinement, first occurs locally at $\rho \simeq 0.3$ and then propagates both inwards and slightly outwards. A fit to experimental data based on a simple temporal-evolution model suggests that the local diffusivity reduction may occur over as short a time as $0.2 \mathrm{~ms}$, one order of magnitude less than $\tau_{e E{ }^{27}}$

The transition occurs during a phase in which all external actuators are held constant. As the primary quantity that is known to evolve continuously during this phase, the current-density profile is likely to have an important role in the formation of the barrier. No current-profile measurements are currently available on $\mathrm{TCV}$, and we must rely instead on a combination of indirect experimental measurements and numerical modeling to assess the current-density distribution and separate its various components.

The Ohmic current-density profile before the auxiliary heating begins can be calculated from Thomson-scattering electron-temperature measurements by assuming neoclassical resistivity. As the temperature is centrally peaked, so is the current density. The noninductively driven current profile in the steady-state phase, in which no inductive component is present, is estimated as follows. The bootstrap component $j_{\mathrm{BS}}$ is calculated from electron density and temperature measurements by Thomson scattering. ${ }^{28}$ From this, the total ECCD current is trivially obtained by subtraction. The ECCD current density profile $j_{\mathrm{EC}}$ can then be calculated by the Fokker-Planck quasilinear code CQL3D, ${ }^{29}$ by adjusting the radial electron diffusivity such as to match the total driven current. ${ }^{30}$ This procedure, which has become standard for TCV, has its origin in the insight gained from experimental and numerical work on the radial transport of suprathermal electrons. Experimental evidence of broadening of the suprathermal-electron spatial distribution goes hand in hand with the observation that the quasilinear enhancement of ECCD efficiency is weaker than expected at the high ECRH power density of TCV: both effects are explained by radial electron transport. ${ }^{31}$ Both $j_{\mathrm{BS}}$ and $j_{\mathrm{EC}}$ are found to be peaked off-axis, as expected. ${ }^{22}$ The noninductive $j$ profiles and the corresponding $q$ profiles are shown in Fig. 7.

Thus at some time between the zeroing of the external electric field and the end of the current redistribution process the $q$ profile must become nonmonotonic. The time-varying phase is more challenging to model, since the CQL3D code does not include the back emf from the plasma. A simplified, intuitive approximate model can be used to gain insight into the role of the current profile and to guide a more rigorous subsequent modeling. This model uses profile form factors and absolute magnitudes for the various current components that are theoretically predicted functions of the measured density and temperature profiles. ${ }^{26}$ By assuming that the Ohmic current decays exponentially, the entire current profile evolution can then be obtained by a fit to the experimental data. The reversal of the $q$ gradient occurs off-axis, with the minimum $q$ location appearing at $\rho \simeq 0.3$. At this time and location, the eITB is formed. This model thus suggests that the barrier is directly tied to the existence of a $s=0$

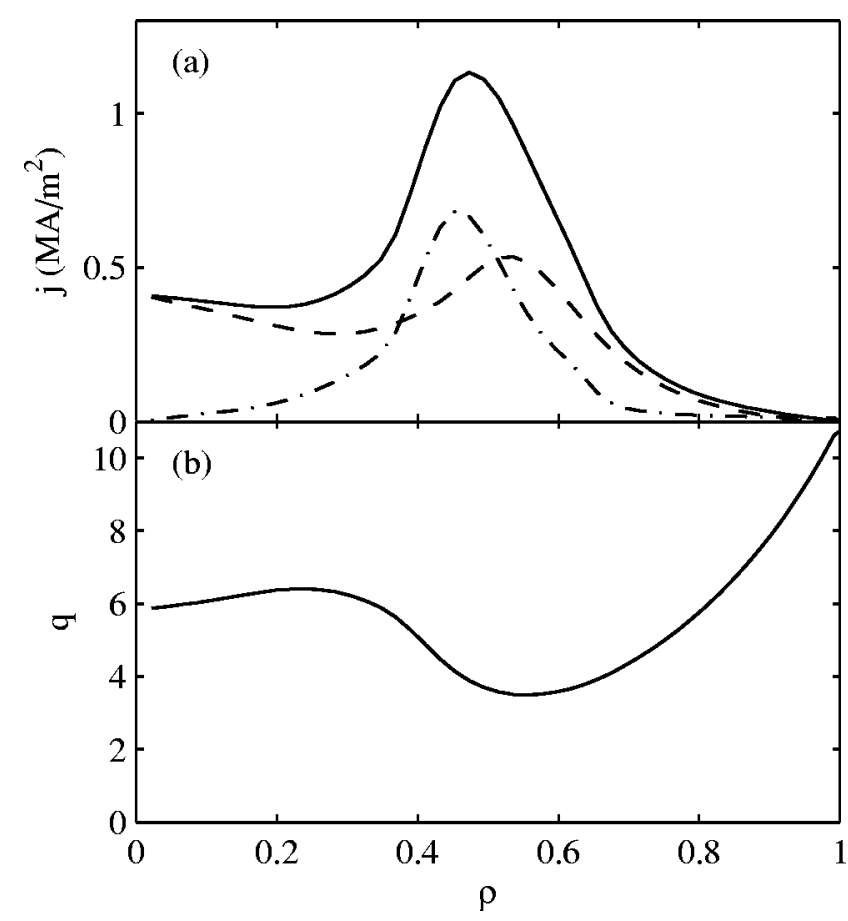

FIG. 7. (a) Current-density profiles: total (solid curve), ECCD component (dashed), bootstrap component (dash dotted); (b) safety factor profile, for TCV discharge 21655 at $1.6 \mathrm{~s}$, in the steady-state eITB phase.

location. ${ }^{26}$ More rigorous simulations using the transport code ASTRA (Ref. 32) to model the current profile evolution are underway and will be the subject of a future publication.

\section{THE STEADY-STATE PHASE OF THE EITB}

The characteristic time for current redistribution $\tau_{\mathrm{CRT}}$ from the initial peaked Ohmic profile to the ultimate hollow noninductive profile is typically of the order of $0.2 \mathrm{~s}$ in the scenarios discussed in this paper. This time can be estimated experimentally by fitting a simple exponential time variation to the evolution of equilibrium parameters such as the internal inductance. The observed time is consistent with simple order-of-magnitude arguments based on resistive diffusion and has also been confirmed by ASTRA simulations. ${ }^{27}$ After the current profile has been allowed to evolve over (2-3) $\times \tau_{\mathrm{CRT}}$, it has reached a steady state. The barrier can be maintained for the whole $2 \mathrm{~s}$ duration of the gyrotron pulse length and is therefore in steady state for up to $8 \times \tau_{\mathrm{CRT}}$.

The eITBs generated in TCV are thus truly stationary in that they are fully noninductively sustained, are maintained at constant total current and with all core plasma parameters stationary, and are finite in duration only because of technical constraints and not because of fundamental physics limits. In particular, the location of the barrier is unvarying throughout the stationary phase, as illustrated by Fig. 8.

The steep electron-pressure gradients that develop once the eITB is formed, particularly after the subsequent application of central heating, tend to drive MHD instabilities that can then degrade the barrier or even cause the plasma to disrupt under some conditions. Ideal MHD calculations using the code KINX (Ref. 33) show that ideal modes can occur near the $q=1, q=2$, and $q=3$ surfaces when the minimum of 


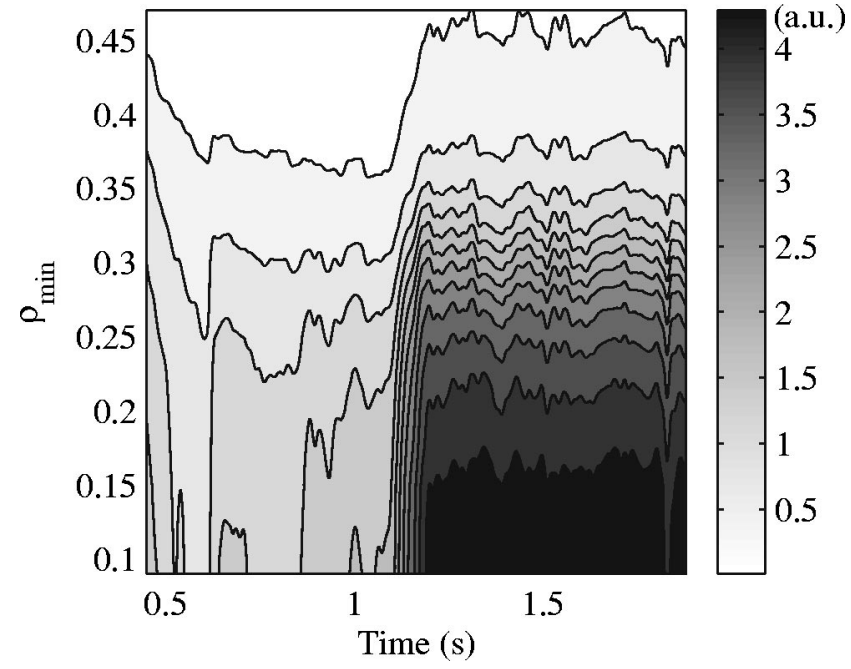

FIG. 8. Contours of vertically line-integrated soft-x-ray emissivity on the low-field side of the torus, as a function of the minimum $\rho$ for each chord, for TCV discharge 21655 .

the safety factor is in the proximity of those values. The global normalized-beta $\left(\beta_{N}=\beta a B_{T} / I_{p}\right)$ limit is effectively reduced under these conditions up to 7 times compared with its $L$-mode level, as shown in Fig. 9 for a case in which the latter is $\beta_{N, \max } \simeq 5.0$. The modes have a predominantly local character, owing to the large local pressure gradient, and have been linked with experimentally observed periodic relaxation oscillations. Additionally, tearing modes can also be destabilized during the eITB; these resistive MHD instabilities, which are readily identified by the analysis of magnetic pick-up coil signals, also cause a reduction in global confinement. ${ }^{34}$

The dependence of these instabilities on the $q$ profile, and particularly on its minimum value, as well as on the pressure gradient, suggests experimental avoidance methods that rely directly on the flexibility of the ECRH sources. The influence of the deposition locations, parallel wave numbers, and power ratio of the different ECRH sources on the properties of the barrier in the steady-state phase has been docu-

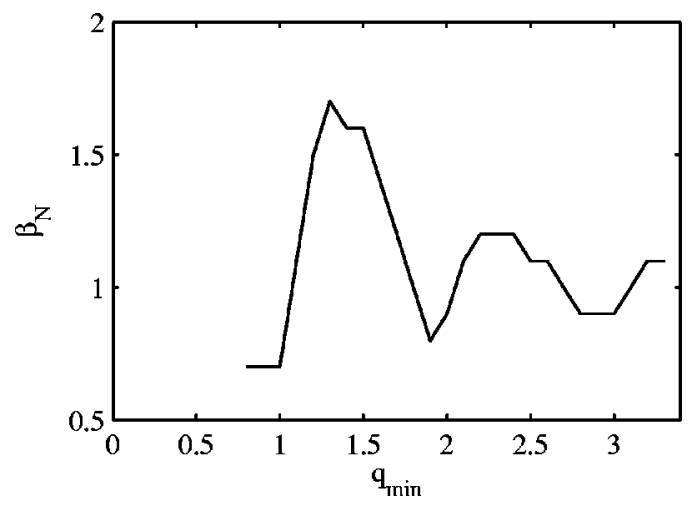

FIG. 9. Stability limit for ideal MHD instability for the TCV eITB discharge 21655, calculated by the KINX code and expressed as an upper limit in normalized $\beta_{N}=\beta a B_{T} / I_{p}(\%, \mathrm{~m}, \mathrm{~T}, \mathrm{MA})$ as a function of the minimum value of the safety factor. The latter is varied by rescaling the total current at constant internal inductance $l_{i}=1.25$. The $\beta_{N}$ limit for conventional scenarios is $\sim 4 l_{i}=5.0$. mented in Ref. 21 . Here we sketch briefly a summary of the principal relations. The location of the off-axis ECRH sources has a strong influence on both the location and the strength $\rho_{\max }^{*}$ of the barrier, which moves with the deposition location and becomes stronger as it shifts outward, within the range in which current drive remains sufficiently efficient. The parallel wave number of the central ECRH beam(s) has a significant effect on the strength, but only a weak one on the position of the eITB. A counter-ECCD component, which also increases $q_{\text {min }}$, tends to strengthen the barrier and vice versa. The power ratio between the central and off-axis sources is an additional control parameter. Experimentally, it has proven feasible to combine these actuators so as to steer a stable course towards the formation of the eITB and the attainment of steady state. The control is robust in that it is reproducible and the safety margin can clearly be ascertained by experimental tuning.

The enhancement parameter $H_{\mathrm{RLW}}$ was found to scale approximately as the strength of the barrier $\rho_{\max }^{*}$ multiplied by the volume enclosed by it. ${ }^{15}$ The dependence of $\rho_{\max }^{*}$ and thus $H_{\mathrm{RLW}}$ on the central current-drive component has special significance as it suggests that an increased negative shear in the core region has a beneficial effect on the overall confinement. However, the barrier appears to be initially created in the $s=0$ region, over a width that is narrower than the ECRH deposition profile, indicating that the negative-shear $(s<0)$ region may not play a role at first; only afterwards does the enhanced confinement extend to the rest of the deposition profile. Additionally, the suddenness of the eITB appearance, discussed in the preceding section, is not compatible with a smooth dependence of $\chi_{e}$ on $s$ as the latter moves from positive to negative. These observations offer clear and strong constraints to theories that attribute the confinement improvement to the suppression of microinstabilities in the presence of weak or negative shear: ${ }^{35,36}$ the dependence on shear must be highly nonlinear. Whether the diffusivity $\chi_{e}$ is significantly lowered throughout the core is not known at present, as no eITB scenario has yet been generated with heating that is strictly on-axis and sufficiently localized. Indeed, simulations suggest that differentiating between a narrow and an extended reduced-transport region may be difficult within the experimental uncertainties because of the intrinsic ECRH beamwidth. ${ }^{37}$

Hence, important open qualitative questions remain, even before the quantitative ones can be addressed about the detailed dependence of both $\chi_{e}$ and $\dot{\chi}_{e}$ on $s$ and $q$ : Does the increased negative central shear affect the barrier at $s=0$ indirectly through a modification of its gradient $s^{\prime}$ there, or does it act to reduce the local energy diffusivity? ${ }^{38-40}$ In the latter case, does the dynamical evolution $\left(\dot{\chi}_{e}\right)$ depend on $s$, resulting in a delayed but ultimately efficient diffusivity quench in the center? If the barrier instead does remain localized at $s=0$, as advocated by the so-called radial-gap or zero-shear-gap theories, ${ }^{41}$ what parameters govern its width? Finally, is there a power flow-threshold for the creation of a barrier? 


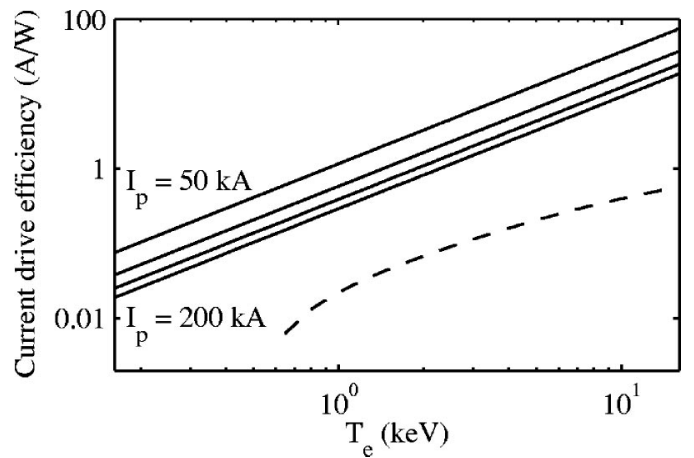

FIG. 10. Theoretical incremental current-drive efficiency for Ohmic drive (solid curves, for total plasma currents ranging from 50 to $200 \mathrm{kA}$ in $50 \mathrm{kA}$ steps) and ECCD (dashed curve) as a function of electron temperature. Neoclassical resistivity has been assumed and the ECCD efficiency is calculated by a linear ray-tracing code for a centrally deposited beam with $30^{\circ}$ angle to the normal to the magnetic field at the resonance. The reference parameters and profiles are from the eITB phase of shot 21655 with $n_{e 0}$ $=1.1 \times 10^{19} \mathrm{~m}^{-3}$.

\section{RESPONSE OF EITBS TO OHMIC CURRENT PERTURBATIONS}

The intrinsically pulsed nature of the transformer action constitutes a significant disadvantage, which has motivated a significant worldwide effort in the development of noninductively driven discharges for potential steady-state operation. ${ }^{42}$ However, the tranformer principle provides by far the most efficient method of driving a toroidal current, measured in terms of driven current per unit dissipated power. A comparison of Ohmic current-drive efficiency with ECCD efficiency is shown in Fig. 10 for typical TCV eITB discharge parameters to illustrate this point. Perturbative Ohmic current injection is therefore an efficient means of adding a small current variation to an otherwise noninductively driven discharge, with negligible energy transfer. This permits, in principle, the study of the effect of a current perturbation in isolation. The steady-state spatial distribution of the perturbed current cannot be controlled independently, as it follows the conductance profile and thus is essentially proportional to $T_{e}^{3 / 2}$. This is, however, ideal for probing the role of the central current in the physics of the eITB. The need to allow for full current penetration to occur is not a particular concern in TCV, given the time scales for resistive diffusion that have been presented in the previous sections. Even in a high-performance eITB scenario, current penetration is essentially complete after $0.5 \mathrm{~s}$ (Ref. 43) (see the following section).

The role of the central current, or equivalently of the $q$ profile inside the barrier, and, in particular, of its steepness in the $s<0$ region, have been evidenced by varying the central current-drive component, as discussed in Sec. III. However, the current source in this case coincides with the main heating source in the core region. Varying the toroidal injection angle of the beam, hence its parallel wave number, does not change the absorbed power appreciably; however, the deposition location and width do change as a result of the varying Doppler shift experienced by the resonant electrons. An Ohmic perturbation experiment, by contrast, offers the pos-

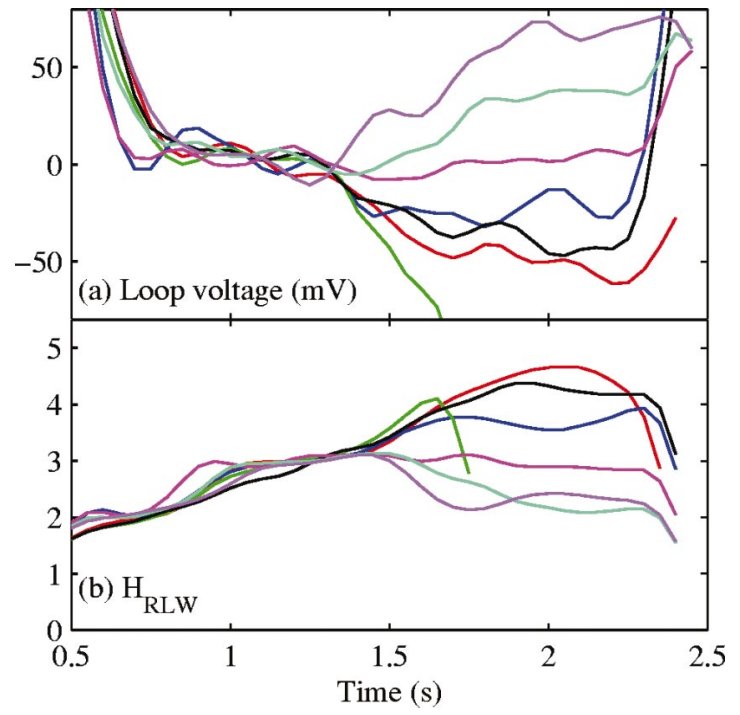

FIG. 11. (Color). (a) Surface loop voltage and (b) electron-energy confinement enhancement factor for different externally applied voltages. The discharge with the most negative voltage (countercurrent) and the largest enhancement became unstable and disrupted.

sibility of a pure, well-controlled central current source which can be varied in sign and magnitude without altering the power deposition profile.

We have carried out such an experiment, using a reference eITB scenario with central ECRH and no central current drive, which does not reach the highest possible stable performance and thus leaves a margin for improvement. A current ramp is preprogramed in the Ohmic transformer primary starting at a time well within the steady-state phase of the discharge. Feedback control is again used on the applied coil voltage to obtain the desired current wave form. One therefore has direct control over the surface loop voltage, which has been varied in steps from -90 to $+60 \mathrm{mV}$ (countercurrent to cocurrent). The result is shown in Fig. 11. Central cocurrent clearly degrades the barrier while countercurrent improves it, as measured by the confinement enhancement. The strength of the barrier follows a similar trend, whereas its position is essentially unaltered, as shown in Fig. 12. The Ohmic power dissipation here is $\sim 3 \mathrm{~kW}$, a truly negligible $0.2 \%$ of the total heating power. Momentum injection is equally negligible. This constitutes unambiguous

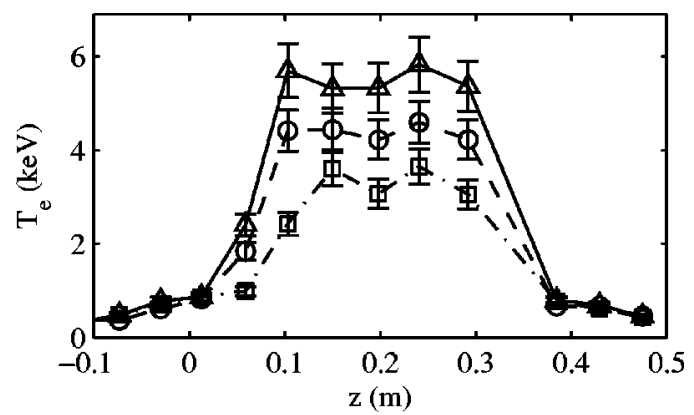

FIG. 12. Electron-temperature profiles for three discharges of the series shown in Fig. 11: 25956 (0 V, circles and dashed curve), 25958 (+60 mV, squares and dash-dotted curve), 25952 ( $-60 \mathrm{mV}$, triangles and solid curve) at time $1.95 \mathrm{~s}$ 


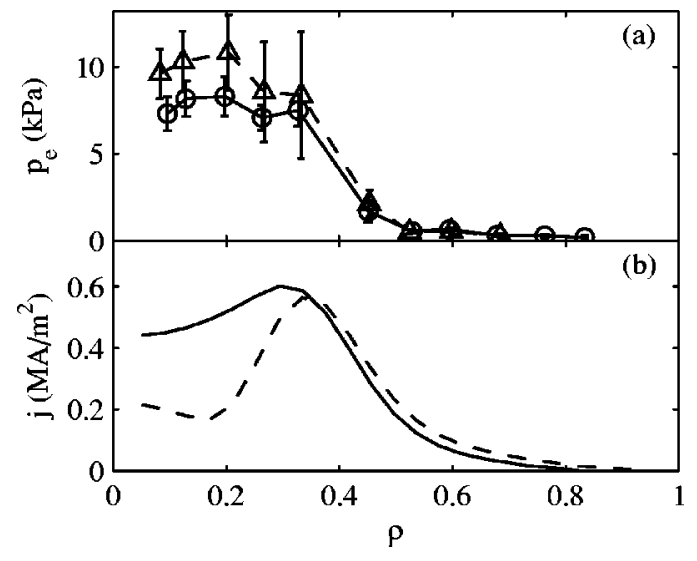

FIG. 13. (a) Electron-pressure profiles averaged from 1.9 to $2.2 \mathrm{~s}$ and (b) current-density profiles calculated by a Fokker-Planck simulation for discharges 25953 (circles and solid curves, $-30 \mathrm{mV}$ loop voltage, $0^{\circ}$ toroidal angle of the central beam) and 25960 (triangles and dashed curves, $0 \mathrm{mV}$ loop voltage, $5^{\circ}$ toroidal angle in the counter-ECCD direction). The simulations are run by keeping the discharge conditions identical using shot 25956 as reference. The confinement enhancement factor $H_{\text {RLW }}$ is $\approx 3.7$ in 25953 and 4.1 in 25960.

proof that the current profile plays a crucial role in determining the confinement properties. ${ }^{24,25}$ It can also be concluded that no threshold exists in the power flow variation required to affect an existing eITB, although the crucial question of whether the formation of the barrier is subject to a power threshold remains open.

No discontinuity or nonlinearity (as could be expected from a bifurcation, e.g.) is observed in this scan. The confinement enhancement $H_{\mathrm{RLW}}$, in particular, varies continuously with the loop voltage, a qualitatively similar dependence to that on the toroidal injection angle, ${ }^{21}$ as is to be expected if the latter is attributed primarily to the change in central driven current. The similarity continues with the modest effect that both actuators have on the position of the eITB. We have run a CQL3D (Fokker-Planck quasilinear) simulation for cases with, respectively, central countercurrent drive with a $5^{\circ}$ angle of the wave vector to the normal to the magnetic field line, and central ECRH (no ECCD) with -30 $\mathrm{mV}$ loop voltage (the off-axis co-ECCD sources are identical in the two cases). The total current calculated by the code is the same in both cases, although the current profile is slightly more hollow in the counter-ECCD case. This is consistent with the slightly better confinement in this case, as evidenced by Fig. 13 .

As a further test of the role of the current profile in these experiments, in the initial current-penetration phase the plasma undergoes a transient confinement evolution that goes opposite to the ultimate state; i.e., a temporary eITB enhancement is seen with positive loop voltage and vice versa. This can be readily understood as, for instance, a positive Ohmic perturbation propagates inward from the edge and initially drives the central shear more negative before rendering it less negative once it has fully penetrated. This dynamic behavior has also been confirmed by ASTRA simulations of the current profile evolution. ${ }^{25,43}$

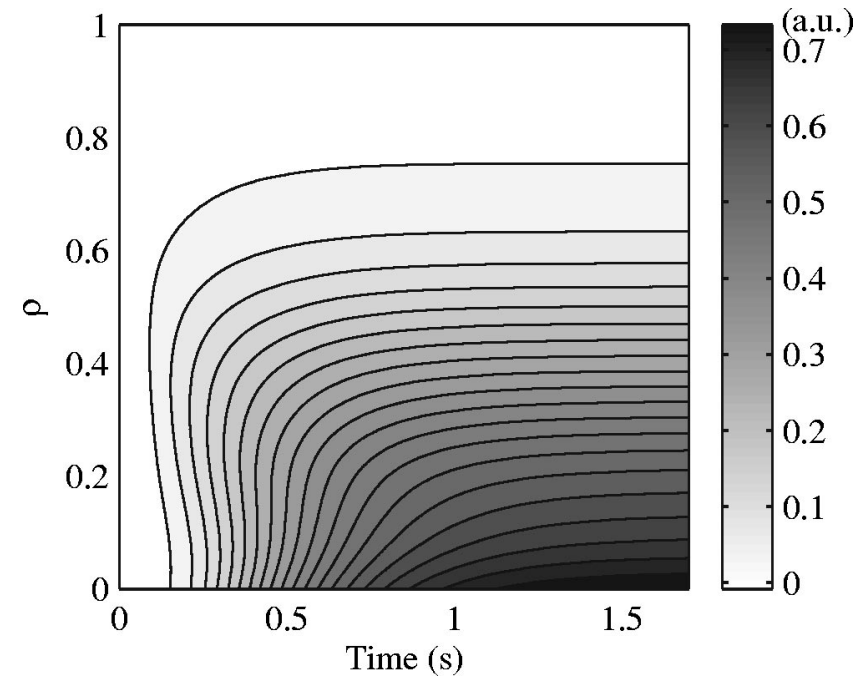

FIG. 14. Spatiotemporal contours of Ohmic current-density profiles calculated by ASTRA in interpretative mode for discharge 25957 with an external voltage perturbation of $+30 \mathrm{mV}$.

\section{MODELING OF EITBS}

Modeling of TCV eITB discharges has recently been performed using the 1.5D transport code ASTRA. ${ }^{32}$ The code includes a system of 1D diffusion equations to describe the density and temperature evolution of the plasma components, a 2D MHD equilibrium solver, and various modules to describe additional heating, current drive, and nondiffusive processes. The electron-cyclotron driven-current profile, in particular, is provided by CQL3D. ASTRA can be employed in both an interpretative mode, with experimentally measured density and temperature profiles or, equivalently, transport coefficients; and in a predictive mode, with theory-based transport coefficients.

Employed in an interpretative mode, ASTRA is a valuable tool for studying the evolution of the current density profile, of which there is no direct measurement. Simulations have confirmed that the safety-factor profile is expected to be reversed in the standard noninductive eITB scenarios discussed in this paper, even if the current driven off-axis by ECCD is taken to be at the low end of the uncertainty attributed to the CQL3D modeling. ${ }^{43}$

An analysis of the Ohmic perturbation experiments described in Sec. IV has also been carried out to model in detail the penetration of the Ohmic perturbation into the plasma core. The evolution of the Ohmic current profile is shown in Fig. 14 for a discharge with $+30 \mathrm{mV}$ (cocurrent) applied voltage. The evolution of the integrated Ohmic current is closely described by an exponential fit with characteristic time $\tau_{\mathrm{CRT}} \simeq 0.15-0.2 \mathrm{~s}$. This time is found to scale as $T_{e}^{3 / 2}$ as expected. ${ }^{43}$

Predictive modeling of the standard eITB scenario has also been carried out by coupling to ASTRA the gyro-Landaufluid code GLF23, ${ }^{44}$ which derives transport coefficients from first principles through a mixing-length modeling based on calculated microinstability growth rates and perpendicular wave numbers. Preliminary results are promising in that the simulation is successful in predicting the formation of a 
barrier. ${ }^{37}$ This is due to a substantial reduction of the theoretical energy diffusivity in the negative-shear region, the effect being maximum in the proximity of the $s=0$ location. The primary physics behind the $\chi_{e}$ suppression in GLF23 is the Shafranov-shift effect on the trapped-electron toroidal precession. ${ }^{40}$ This effect can be artificially turned off in the code, in which case no significant suppression is predicted. Global linear gyrokinetic simulations have confirmed that trapped-electron modes are expected to be the dominant microinstability in these TCV discharges; these simulations also show a reduction in the mixing-length diffusivity associated with low- $n$ modes, which tend to be localized around the barrier location. ${ }^{45}$ This again is consistent with a reduction in cross-field transport.

\section{CONCLUSIONS}

Work on improved core-confinement regimes in TCV has focused in the past few years on achieving steady-state conditions, building on an established program of fully noninductive tokamak operation using ECCD. Highperformance regimes with bootstrap fraction up to $75 \%$, electron energy confinement enhancement factor up to 5 , and poloidal $\beta$ up to 2.4 have been obtained in stationary conditions for durations several times longer than the characteristic current relaxation time and limited only by technological constraints. These discharges feature steep electron pressure gradients at $1 / 3$ to $1 / 2$ of the minor radius, which are termed electron internal transport barriers.

Strong evidence ties the barrier to the existence of a nonmonotonic safety-factor profile, with a core region of negative shear. Although TCV has no direct current-profile measurement at present, interpretative transport simulations have been used to model the evolution of the current profile, with constraints both from experimental measurements and from quasilinear current-drive calculations, and have indicated that the safety factor is indeed nonmonotonic in all the eITB scenarios studied. The role of the current profile has been studied in isolation through the application of a novel technique consisting of Ohmic current perturbations to an otherwise fully noninductive discharge. Small increases or decreases in the core negative shear, without any associated energy transfer, cause substantial enhancements or degradations of the confinement, respectively. This observation is a more controlled version of a similar experiment using different degrees of countercurrent drive in the center, and both point to a direct dependence of the barrier quality on the central shear. However, the initial barrier formation itself has been found to be a localized event at the location of the minimum $q$, with the confinement improvement later extending to a broader region, although it is not yet known how broad this region is on the inboard side. Additionally, the barrier creation is a sudden event occurring over a confinement time scale or less at the time of shear reversal, suggesting that the transition from a monotonic to a nonmonotonic $q$ profile causes a nonlinear jump in the transport coefficients, presumably related to a drastic suppression of local turbulence.

Numerical simulations of microinstability evolution sup- port the conclusion that turbulence and turbulent transport are strongly reduced in the proximity of the minimum $q$ region. The dynamical evolution leading to the formation of the barrier, and the possible nonlinearities or discontinuities associated with it, however remains to be studied. A number of questions thus remain open, particularly concerning the level of transport in the region enclosed by the location with the steepest gradient, concerning the connection between the central shear and the energy diffusivity at the barrier, and concerning the temporal dynamics of the transport quench. While the results are thus insufficient to permit a clear choice between existing theories, they do, however, strongly constrain the theoretical efforts to come.

\section{ACKNOWLEDGMENT}

This work was supported in part by the Swiss National Science Foundation.

\section{APPENDIX: THE TCV TEAM}

S. Alberti, P. Amorim, ${ }^{\text {a }}$ Y. Andrèbe, K. Appert, G. Arnoux, R. Behn, A. Bortolon, A. Bottino, Y. Camenen, R. Chavan, S. Coda, I. Condrea, B. P. Duval, P. Etienne, E. Fable, D. Fasel, A. Fasoli, T. P. Goodman, B. Gulejová, M. A. Henderson, J.-P. Hogge, J. Horacek, P.-F. Isoz, B. Joye, A. Karpushov, I. Klimanov, P. Lavanchy, J. B. Lister, X. Llobet, T. Madeira, ${ }^{a}$ J.-C. Magnin, A. Marinoni, J. Márki, B. Marlétaz, P. Marmillod, Y. Martin, An. Martynov, M. Maslov, J.-M. Mayor, J.-M. Moret, A. Mück, P. Nikkola, P. J. Paris, I. Pavlov, A. Perez, R. A. Pitts, A. Pochelon, L. Porte, O. Sauter, A. Scarabosio, Ch. Schlatter, K. Schombourg, H. Shidara, M. Siegrist, U. Siravo, A. V. Sushkov, ${ }^{\text {b }}$ G. Tonetti, M. Q. Tran, H. Weisen, M. Wischmeier, A. Zabolotsky, G. Zhuang, C. Zucca.

${ }^{\text {a }}$ Associação EURATOM/IST, Centro de Fusão Nuclear, Av. Rovisco Pais, 1049-001 Lisbon, Portugal.

b Nuclear Fusion Institute, RRC Kurchatov Institute, 123182 Moscow, Russian Federation.

${ }^{1}$ F. Wagner, G. Becker, K. Behringer et al., Phys. Rev. Lett. 49, 1408 (1982).

${ }^{2}$ J. W. Connor, T. Fukuda, X. Garbet, C. Gormezano, V. Mukhovatov, M. Wakatani, the ITB Database Group, and the ITPA Topical Group on Transport and Internal Barrier Physics, Nucl. Fusion 44, R1 (2004), and references therein.

${ }^{3}$ K. H. Burrell, Phys. Plasmas 6, 4418 (1999).

${ }^{4}$ T. Ohkawa, Nucl. Fusion 10, 185 (1970).

${ }^{5}$ W. A. Houlberg and S. E. Attenberger, Fusion Technol. 26, 566 (1994).

${ }^{6}$ X. Litaudon, R. Arslanbekov, G. T. Hoang et al., Plasma Phys. Controlled Fusion 38, 1603 (1996).

${ }^{7}$ G. M. D. Hogeweij, N. J. Lopes Cardozo, M. R. De Baar, and A. M. R. Schilham, Nucl. Fusion 38, 1881 (1998).

${ }^{8}$ ITER Physics Expert Group on Confinement and Transport, ITER Physics Expert Group on Confinement Modelling and Database, and ITER Physics Basis Editors, Nucl. Fusion 39, 2175 (1999).

${ }^{9}$ S. Alberti, A. Arnold, E. Borie et al., Fusion Eng. Des. 53, 387 (2001).

${ }^{10}$ Y. Koide and the JT-60 Team, Phys. Plasmas 4, 1623 (1997).

${ }^{11}$ S. Coda, T. P. Goodman, M. A. Henderson et al., Plasma Phys. Controlled Fusion 42, B311 (2000).

${ }^{12}$ E. J. Doyle, G. M. Staebler, L. Zeng et al., Plasma Phys. Controlled Fusion 42, A237 (2000).

${ }^{13}$ F. Hofmann, J. B. Lister, M. Anton et al., Plasma Phys. Controlled Fusion 36, B277 (1994).

${ }^{14}$ Z. A. Pietrzyk, C. Angioni, R. Behn, S. Coda, T. P. Goodman, M. A. 
Henderson, F. Hofmann, and O. Sauter, Phys. Rev. Lett. 86, 1530 (2001). ${ }^{15}$ M. A. Henderson, S. Alberti, C. Angioni et al., Phys. Plasmas 10, 1796 (2003).

${ }^{16}$ T. P. Goodman, S. M. Ahmed, S. Alberti et al., Nucl. Fusion 43, 1619 (2003).

${ }^{17}$ O. Sauter, M. A. Henderson, F. Hofmann et al., Phys. Rev. Lett. 84, 3322 (2000).

${ }^{18}$ O. Sauter, C. Angioni, S. Coda et al., Phys. Plasmas 8, 2199 (2001).

${ }^{19}$ O. Sauter, R. Behn, S. Coda, I. Condrea, T. P. Goodman, M. A. Henderson, and P. Nikkola, Proceedings of the 29th EPS Conference on Plasma Physics and Controlled Fusion, Montreux, 2002, edited by R. Behn and C. Varandas (European Physical Society, Mulhouse, 2002); Europhys. Conf. Abstr. 26B, P-2.087 (2002).

${ }^{20}$ P. H. Rebut, P. P. Lallia, and M. L. Watkins, Proceedings of the 12th International Conference on Plasma Physics and Controlled Fusion Research, Nice, 1988 (IAEA, Vienna, 1989), Vol. 2, p. 191.

${ }^{21}$ M. A. Henderson, R. Behn, S. Coda et al., Plasma Phys. Controlled Fusion 46, A275 (2004).

${ }^{22}$ P. Nikkola, Ph.D. thesis, Ecole Polytechnique Fédérale de Lausanne, No. 3048, Lausanne, Switzerland, 2004.

${ }^{23}$ O. Sauter, R. Behn, P. Bosshard, S. Coda, I. Condrea, T. P. Goodman, M. A. Henderson, and P. Nikkola, Proceedings of the 19th IAEA Fusion Energy Conference, Lyon, 2002 (IAEA, Vienna, 2002), EXP/P5-6.

${ }^{24}$ T. P. Goodman, R. Behn, A. Bottino, S. Coda, E. Fable, M. A. Henderson, P. Nikkola, O. Sauter, and C. Zucca, in Proceedings of the 31st EPS Conference on Plasma Physics and Controlled Fusion, London, 2004, edited by P. Norreys and H. Hutchinson (European Physical Society, Mulhouse, 2004); Europhys. Conf. Abstr. 28G, O-2.05 (2004).

${ }^{25}$ O. Sauter, S. Coda, T. P. Goodman, M. A. Henderson, R. Behn, A. Bottino, E. Fable, An. Martynov, P. Nikkola, C. Zucca, and the TCV team, Phys. Rev. Lett. 94, 105002 (2005).

${ }^{26}$ M. A. Henderson, Y. Camenen, S. Coda, T. P. Goodman, P. Nikkola, A. Pochelon, O. Sauter, and the TCV team, Phys. Rev. Lett. 93, 215001 (2004).

${ }^{27}$ M. A. Henderson, Y. Camenen, S. Coda, E. Fable, T. P. Goodman, P. Nikkola, A. Pochelon, O. Sauter, C. Zucca, and the TCV team, Proceedings of the 20th IAEA Fusion Energy Conference, Vilamoura, 2004 (IAEA, Vienna, 2004), EXP/P3-3.

${ }^{28}$ O. Sauter, C. Angioni, and Y. R. Lin-Liu, Phys. Plasmas 6, 2834 (1999); 9, 5140(E) (2002).

${ }^{29}$ R. W. Harvey and M. G. McCoy, Proceedings of the IAEA Technical Committee Meeting on Advances in Simulation and Modeling in Thermo- nuclear Plasmas, Montreal, 1992 (IAEA, Vienna, 1993), p. 498.

${ }^{30}$ P. Nikkola, O. Sauter, R. Behn, S. Coda, I. Condrea, T. P. Goodman, M. A. Henderson, R. W. Harvey, and the TCV Team, Nucl. Fusion 43, 1343 (2003).

${ }^{31}$ S. Coda, S. Alberti, P. Blanchard, T. P. Goodman, M. A. Henderson, P. Nikkola, Y. Peysson, and O. Sauter, Nucl. Fusion 43, 1361 (2003).

${ }^{32}$ G. V. Pereverzev, F. X. Söldner, R. Bartiromo, F. Leuterer, and V. V. Parail, Nucl. Fusion 32, 1023 (1992).

${ }^{33}$ L. Degtyarev, A. Martynov, S. Medvedev, F. Troyon, L. Villard, and R. Gruber, Comput. Phys. Commun. 103, 10 (1997).

${ }^{34}$ G. Zhuang, A. Scarabosio, R. Behn, Y. Camenen, T. P. Goodman, M. A. Henderson, I. Klimanov, A. Martynov, O. Sauter, H. Weisen, and the TCV team, Proceedings of the 31st EPS Conference on Plasma Physics and Controlled Fusion, London, 2004, edited by P. Norreys and H. Hutchinson (European Physical Society, Mulhouse, 2004); Europhys. Conf. Abstr. 28G, P2.143 (2004)

${ }^{35}$ X. Garbet, C. Bourdelle, G. T. Hoang, P. Maget, S. Benkadda, P. Beyer, C. Figarella, I. Voitsekovitch, O. Agullo, and N. Bian, Phys. Plasmas 8, 2793 (2001).

${ }^{36}$ J. Candy, R. E. Waltz, and M. N. Rosenbluth, Phys. Plasmas 11, 1879 (2004).

${ }^{37}$ E. Fable and O. Sauter, Theory of Fusion Plasmas, Proceedings of the Joint Varenna-Lausanne International Workshop, Varenna, 2004, edited by J. W. Connor, O. Sauter, and E. Sindoni (Società Italiana di Fisica, Bologna, 2004), p. 443.

${ }^{38}$ R. E. Waltz, G. D. Kerbel, J. Milovich, and G. W. Hammett, Phys. Plasmas 2, 2408 (1995).

${ }^{39}$ J. F. Drake, Y. T. Lau, P. N. Guzdar, A. B. Hassam, S. V. Novakovski, B. Rogers, and A. Zeiler, Phys. Rev. Lett. 77, 494 (1996).

${ }^{40}$ M. Beer, G. W. Hammett, G. Rewoldt, E. J. Synakowski, M. C. Zarnstorff, and W. Dorland, Phys. Plasmas 4, 1792 (1997).

${ }^{41}$ Y. Kishimoto, J.-Y. Kim, W. Horton, T. Tajima, M. J. LeBrun, S. A. Dettrick, J. Q. Li, and S. Shirai, Nucl. Fusion 40, 667 (2000).

${ }^{42}$ X. Litaudon, Plasma Phys. Controlled Fusion 40, A251 (1998).

${ }^{43}$ C. Zucca, E. Fable, P. Nikkola, and O. Sauter, Theory of Fusion Plasmas, Proceedings of the Joint Varenna-Lausanne International Workshop, Varenna, 2004, edited by J. W. Connor, O. Sauter, and E. Sindoni (Società Italiana di Fisica, Bologna, 2004), p. 449.

${ }^{44}$ R. E. Waltz, G. M. Staebler, W. Dorland, G. W. Hammett, M. Kotschenreuther, and J. A. Konings, Phys. Plasmas 4, 2482 (1997).

${ }^{45}$ A. Bottino, Ph.D. thesis, Ecole Polytechnique Fédérale de Lausanne, No. 2938, Lausanne, Switzerland, 2004. 